\title{
Identification of Akt1 as a potent therapeutic target for oral squamous cell carcinoma
}

\author{
KOH-ICHI NAKASHIRO ${ }^{1}$, HIROSHI TANAKA ${ }^{1}$, HIROYUKI GODA ${ }^{1}$, KAZUKI IWAMOTO ${ }^{1}$, NORIHIKO TOKUZEN ${ }^{1}$, \\ SHINGO HARA $^{2}$, JUN ONODERA ${ }^{3}$, ICHIRO FUJIMOTO ${ }^{3}$, SATOSHI HINO $^{1}$ and HIROYUKI HAMAKAWA ${ }^{1}$ \\ ${ }^{1}$ Department of Oral and Maxillofacial Surgery, Ehime University Graduate School of Medicine, \\ Toon, Ehime; ${ }^{2}$ Department of Oral and Maxillofacial Surgery, Kochi Health Sciences Center, \\ Kochi, Kochi; ${ }^{3}$ Koken Research Institute, Koken Co., Ltd., Tokyo, Japan
}

Received June 16, 2015; Accepted August 3, 2015

DOI: $10.3892 /$ ijo.2015.3134

\begin{abstract}
Oncogene addiction can provide therapeutic opportunities in human malignancies. In this study, we aimed to identify critical oncogenes for oral squamous cell carcinoma (OSCC) development and progression. We determined gene expression profiles in 10 primary OSCCs and 10 human OSCC cell lines using Applied Biosystems Human Genome Survey Arrays. Aktl was the only gene identified that was expressed in all OSCC tissues and cultured cells, but not in non-neoplastic tissues and cells. Subsequently, western blot analysis showed that Akt1 protein was overexpressed in OSCC tissues and cell lines. Immunohistochemistry also showed Akt1 protein expression in 59 of 63 (94\%) primary OSCCs. To clarify the oncogenic function of Akt1 in human OSCC cells, we used RNA interference. We designed and synthesized 5 small interfering RNAs specific for Akt1 (siAkt1). Transfecting human OSCC cells with siAkt1 in vitro markedly suppressed their expression of Akt1 protein and significantly reduced their growth rate. Furthermore, the growth of human OSCC tumors which had been subcutaneously xenografted in athymic nude mice lacking interferon responses was markedly inhibited by atelocollagen-mediated systemic siAktl administration. We also found that synthetic siAkt1 had an inhibitory effect on the growth of primary cultured OSCC cells. Finally, we investigated the molecular mechanisms involved in the growth inhibitory effect of Akt1 suppression using microarray analysis of human OSCC
\end{abstract}

Correspondence to: Dr Koh-Ichi Nakashiro, Department of Oral and Maxillofacial Surgery, Ehime University Graduate School of Medicine, 454 Shitsukawa, Toon, Ehime 791-0295, Japan

E-mail: nakako@m.ehime-u.ac.jp

Abbreviations: HNSCC, head and neck squamous cell carcinoma; OSCC, oral squamous cell carcinoma; siAkt1, small interfering RNA specific for Akt1

Key words: oral squamous cell carcinoma, Akt1, molecular target therapy cells transfected with siAkt1. Knockdown of Akt1 induced the expression of CDKN2B, a tumor suppressor gene, and reduced the expression of TGFBR1, which supports malignant phenotypes. These results suggest that Akt1 functions as a critical oncogene in human OSCC cells and may therefore be an appropriate target for novel OSCC therapies.

\section{Introduction}

More than half a million new cases of head and neck squamous cell carcinoma (HNSCC) occurred in 2008 worldwide. Oral squamous cell carcinoma (OSCC) is the most common HNSCC neoplasia, with over a quarter of a million new cases reported in 2008 with a mortality rate of $\leq 50 \%$ (1). Despite advances in our knowledge of the disease and in chemotherapy, radiotherapy and surgery, little improvement in the relative survival has been seen in OSCC during the past several decades (2). Therefore, a greater understanding of the pathogenesis of OSCC is needed for the development of more effective therapeutic approaches.

Cancer cells acquire abnormalities in multiple oncogenes and tumor suppressor genes. Overexpression and constitutive activation of several oncogenes support the proliferation, invasion, and metastasis of cancer cells. However, inactivation of a single critical oncogene can induce their terminal differentiation or apoptosis and this has suggested that this 'oncogene addiction' is an Achilles heel for cancers, providing therapeutic opportunities in many human malignancies (3). In HNSCC, including OSCC, the overexpression of epidermal growth factor receptor (EGFR) has been shown to be correlated with lymph node or distant metastasis, risk of locoregional recurrence and poor prognosis (4). Cetuximab, which targets EGFR, is used to treat patients with local advanced, recurrent or metastatic HNSCC. Therapies combining radiotherapy or platinum-based chemotherapy with cetuximab have improved locoregional control and overall survival, and reduced mortality $(5,6)$. However, cetuximab is the only molecularly targeted drug available for OSCC patients. In this study, we have attempted to identify other target molecules by microarray analysis and to determine whether targeting such molecules could provide novel, potential therapeutic approaches for the treatment of OSCC patients. 


\section{Materials and methods}

Cells and cell culture. In this study, we used 10 human oral cancer cell lines, which were green fluorescent protein (GFP)-SAS (7), Ca9-22, HSC2, HSC3, HSC4, KB, SCC111, SCC66, SCC9 and SCC25, and an immortalized non-neoplastic human keratinocyte cell line, $\mathrm{HaCaT}$, as described previously $(8,9)$. These cells were grown in Dulbecco's modified Eagle's medium (DMEM; Wako, Osaka, Japan) supplemented with $10 \%$ fetal bovine serum (FBS; Biosource, Camarillo, CA, USA), $100 \mathrm{U} / \mathrm{ml}$ penicillin and $100 \mu \mathrm{g} / \mathrm{ml}$ streptomycin (Wako), referred to here as complete medium.

Primary cell cultures were established from patients' OSCC tumors. After surgical excision, tumor tissue was rinsed several times with complete medium, cut into small fragments and dissociated with $0.1 \%$ collagenase (Wako) at $37^{\circ} \mathrm{C}$ for $2 \mathrm{~h}$. Cell suspensions were filtered through a $70-\mu \mathrm{m}$ nylon mesh cell strainer (BD, Franklin Lakes, NJ, USA). The cells were collected by centrifugation, resuspended in keratinocyte serum-free medium (K-SFM; Life Technologies, Carlsbad, CA, USA), and plated on a plastic surface to grow. All cells were incubated in a humidified atmosphere of $95 \%$ air and $5 \% \mathrm{CO}_{2}$ at $37^{\circ} \mathrm{C}$.

Samples from patients. OSCC and normal oral mucosa epithelial tissues were obtained from patients at the Ehime University Hospital between June 2009 and December 2012. Four primary OSCC cultured cells were established, two from the tongue (a 70-year-old male with a T4N2cM0 stage tumor and a 40-year-old male with a T2N0M0 stage tumor) and two from the gingiva (a 66-year-old male with a T4aNOM0 stage tumor and an 80-year-old female with a T2NOM0 stage tumor). Clinical stages were defined according to the tumornode-metastasis (TNM) classification of malignant tumors (Union Internationale Contre le Cancer). The Institutional Review Board at Ehime University Hospital approved this study (no. 0904009), and appropriate written informed consent was obtained from each patient.

Microarray analysis. To identify oncogenic genes in OSCC, the Applied Biosystems Chemiluminescent RT-IVT Labeling kit (Life Technologies) was used to convert total RNA to digoxigenin (DIG)-labeled cRNA. Total RNA was extracted by lysing the cells or tissues using ISOGEN (NipponGene, Tokyo, Japan). Tissues were homogenized in $0.5 \mathrm{ml}$ ISOGEN using TissueLyser (Qiagen, Valencia, CA, USA). Double-stranded cDNA was generated from $1 \mu \mathrm{g}$ total RNA, transcribed using DIG-labeled nucleotides (Roche Diagnostics, Basel, Switzerland), fragmented, and hybridized to Human Genome Survey Arrays (Life Technologies) according to the manufacturer's instructions. After washing each array, the signal was developed using a chemiluminescent detection kit (Life Technologies). Processed arrays were scanned with a 1700 chemiluminescent microarray analyzer (Life Technologies).

We clarified the molecular mechanisms of the growth inhibitory effects using synthetic small interfering RNA (siRNA) specific for Akt1 (siAkt1). Total RNA was extracted from GFP-SAS cells treated with either $10 \mathrm{nM}$ siAkt1 or nontargeting siRNA (siNT), 100 ng total RNAs were labeled using a GeneAtlas ${ }^{\mathrm{TM}}$ 3'IVT Express kit assay (Affymetrix, Santa
Clara, CA, USA), and hybridized onto Affymetrix Human Genome U219 Array Strips (Affymetrix), which included $>530,000$ probes covering over 36,000 transcripts and variants, according to the manufacturer's instructions. After washing and staining the array strips, they were scanned using the GeneAtlas ${ }^{\mathrm{TM}}$ System (Affymetrix).

These results were analyzed using the GeneSpring GX 12.1 (Agilent Technologies, Santa Clara, CA, USA) and Ingenuity Pathway Analysis software (IPA; Ingenuity ${ }^{\circledR}$ Systems, www. ingenuity.com, Redwood City, CA, USA). Functional analysis with IPA software identified the biological functions and diseases that were most significant in our data set. Fischer's exact test was used to calculate p-values for the probability that each biological function or disease assigned to a data set was due to chance alone. The raw microarray data have been deposited in Gene Expression Omnibus (GEO, http:// www.ncbi.nlm.nih.-gov/geo, experiment nos. GSE36090 and GSE56233) according to the minimum information about microarray experiment (MIAME) guidelines.

Western blot analysis. Cells were grown in monolayers for $48 \mathrm{~h}$ and lysed with lysis buffer of $0.5 \mathrm{M}$ EDTA and $1 \%$ Triton X-100 (Sigma-Aldrich, St. Louis, MO, USA) in phosphate-buffered saline (PBS) containing a protease inhibitor cocktail and phosphatase inhibitor (Roche Diagnostics). Tissues were homogenized in $500 \mu \mathrm{l}$ lysis buffer using a TissueLyser (Qiagen). The samples were centrifuged at $15,000 \mathrm{x} \mathrm{g}$ for $15 \mathrm{~min}$ at $4^{\circ} \mathrm{C}$, and the supernatants were electrophoresed on SDS-polyacrylamide gels and transferred to polyvinylidene difluoride membranes (Millipore, Bedford, MA, USA). The membranes were blocked with 5\% non-fat dried milk (Wako) in $25 \mathrm{mM}$ Tris-HCl, $125 \mathrm{mM} \mathrm{NaCl}$, and $0.1 \%$ Tween-20 (TBS-T; Sigma-Aldrich) for $1 \mathrm{~h}$ at room temperature. They were then probed with monoclonal rabbit anti-human Akt1 antibody (Cell Signaling Technology, Danvers, MA, USA) or monoclonal mouse anti- $\beta$-tubulin antibody (BD) at a 1:1,000 dilution in 5\% non-fat dried milk in TBS-T for $1 \mathrm{~h}$ at room temperature, followed by horseradish peroxidase-conjugated secondary antibodies against rabbit or mouse IgG (GE Healthcare, Little Chalfont, UK) for $1 \mathrm{~h}$ at room temperature. The immune complexes were visualized using the enhanced chemiluminescence (ECL) Prime Western Blotting Detection Reagent (GE Healthcare). The density of the visualized immune complexes was digitized using RAS3000 (Fujifilm, Tokyo, Japan).

Immunohistochemistry. The surgically resected OSCC specimens were fixed in phosphate-buffered $10 \%$ formalin and embedded in paraffin. A series of $4-\mu \mathrm{m}$ thick tissue sections were prepared from each sample. Immunohistochemical staining was performed using the avidin-biotin-peroxidase complex method. Briefly, the sections were deparaffinized, pretreated with $10 \mathrm{mM}$ citrate buffer $\left(\mathrm{pH} \mathrm{6.0)}\right.$ at $95^{\circ} \mathrm{C}$ for $10 \mathrm{~min}$, cooled at room temperature for $30 \mathrm{~min}$, and incubated with $0.3 \% \mathrm{H}_{2} \mathrm{O}_{2}$ in distilled water for 10 min to block endogenous peroxidase activity. After blocking with $3 \%$ horse serum in PBS, the sections were incubated overnight at $4^{\circ} \mathrm{C}$ with a 1:400 dilution of a monoclonal rabbit antibody specific for human Akt1 (Cell Signaling Technology). After washing, the sections were overlaid with biotinylated anti-rabbit anti- 
body (Vector Laboratories, Burlingame, CA, USA) at room temperature for $60 \mathrm{~min}$, washed in PBS, and then labeled with streptavidin-peroxidase complex (Vector Laboratories). The peroxidase reaction was developed with 3'3-diaminobenzidine as a chromogen. The sections were counterstained with hematoxylin, dehydrated with ethanol, treated with xylene, and enclosed in synthetic resin. Negative controls omitted the primary antibody. Cases were considered to be Akt1-positive if the cell nuclei and cytoplasm of $>50 \%$ of the cancer cells were labeled.

Design and transfection of siRNA. We designed and synthesized five siAkt1s. The target sequences were optimized for maximum target-gene silencing, minimum sequencespecific cross reactivity (off-target effects) and avoidance of single nucleotide polymorphisms (SNPs). Synthetic siRNA targeting GFP (siGFP) or siNT were used as negative controls. Transfection was performed using Lipofectamine RNAiMAX (Life Technologies) mixed with $10 \mathrm{nM}$ siRNAs for western blotting and the cell growth assays. The sequences of synthetic siRNAs used were as follows: siAkt1-6: 5'-GAG CGG GAG GAG UGG ACA ATT-3' (sense) and 5'-UUG UCC ACU CCU CCC GCU CTT-3' (antisense); siAkt1-22: 5'-CCA UGA AGA UCC UCA AGA ATT-3' (sense) and 5'-UUC UUG AGG AUC UUC AUG GTT-3' (antisense); siAkt1-33: 5'-CCA AGG AGA UCA UGC AGC ATT-3' (sense) and 5'-UGC UGC AUG AUC UCC UUG GTT-3' (antisense); siAkt1-56: 5'-GGG UUU ACC CAG UGG GAC ATT-3' (sense) and 5'-UGU CCC ACU GGG UAA ACC CTT-3' (antisense); siAkt1-58: 5'-GGA CAG AGG AGC AAG GUU UTT-3' (sense) and 5'-AAA CCU UGC UCC UCU GUC CTT-3' (antisense); siGFP: 5'-CUA CAA CAG CCA CAA CGU CTT-3' (sense) and 5'-GAC GUU GUG GCU GUU GUA GTT-3' (antisense); siNT: 5'-UAC GUA CUA UCG CGC GGA UTT-3' (sense) and 5'-AUC CGC GCG ATA GUA CGU ATT-3' (antisense).

Cell growth assay. Cells were seeded into 96-well plates in complete medium with $10 \mathrm{nM}$ synthetic siRNA and $0.2 \%$ Lipofectamine RNAiMAX in a final volume of $100 \mu 1$. Cell growth was evaluated after $72 \mathrm{~h}$ using WST-8 assays (Cell Counting Kit-8; Dojindo, Kumamoto, Japan).

Three-dimensional collagen-gel assay. Eight volumes of rat tail type I collagen suspension (BD) were mixed with one volume of 10-fold concentrated DMEM (Sigma-Aldrich) and one volume of reconstruction buffer $\left(2.2 \mathrm{~g} \mathrm{NaHCO}_{3}\right.$ and $4.77 \mathrm{~g}$ HEPES in $100 \mathrm{ml}$ of $0.05 \mathrm{~N} \mathrm{NaOH}$; Sigma-Aldrich). The collagen gel and carcinoma cells $\left(5 \times 10^{4}\right.$ cells/well) transfected with each siRNA were together poured into a 24 -well plate $\left(0.5 \mathrm{ml} /\right.$ well). After incubation for $30 \mathrm{~min}$ at $37^{\circ} \mathrm{C}$ to permit complete gelation, DMEM complete medium was added; the medium was changed every other day. After incubation for 4 days, the cells contained within the gel were recovered by treatment with $0.1 \%$ collagenase and $0.5 \%$ trypsin-5.3 mM EDTA (Invitrogen). The cells were counted with a Z1 Coulter ${ }^{\circledR}$ particle counter (Beckman Coulter, Fullerton, CA, USA).

Xenograft model and tumor therapy. GFP-SAS cells $\left(1 \times 10^{6} \%\right.$ site) complexed with Matrigel (BD) in 100- $\mu$ l aliquots were injected subcutaneously at two sites in the flanks of male athymic nude mice (CLEA Japan, Tokyo, Japan). Two weeks later, tumor-bearing nude mice were randomly divided into siGFP, siAkt1-22 or siAkt1-58 treatment groups. Complexes of $40 \mu \mathrm{M}$ siRNAs with atelocollagen (AteloGene; Koken, Tokyo, Japan) were injected into mouse tail veins every 3 days (10-14). Tumor diameters were measured at regular intervals with digital calipers, and tumor volumes $\left(\mathrm{mm}^{3}\right)$ were calculated using the following formula: length $\mathrm{x}$ width $\mathrm{x}$ height $\mathrm{x} 0.523$. Three mice were used in each group. Four weeks after the first administration of siRNAs, the mice were sacrificed humanely, the GFP-SAS xenografts, lungs, livers and kidneys were dissected, and Akt1 protein expression levels were determined by western blotting. The animal studies were approved by the Ehime University Animal Care Committee (no. MA-17-14).

Real-time quantitative reverse transcriptase polymerase chain reaction ( $q R T-P C R)$. Total RNA was extracted from tissues by homogenization in $0.5 \mathrm{ml}$ ISOGEN (NipponGene) using a TissueLyser (Qiagen). The relative mRNA levels were quantified using the comparative $\mathrm{C}_{\mathrm{T}}$ method $\left(\Delta \Delta \mathrm{C}_{\mathrm{T}}\right.$ method) by qRT-PCR using the TaqMan ${ }^{\circledR}$ system. Hydroxymethylbilane synthase (HMBS) was used as an internal control. PCR amplification was performed in a $10-\mu$ l final reaction mixture containing $5 \mu \mathrm{l}$ 2X Quantitect RT-PCR Master Mix, $0.1 \mu \mathrm{l}$ Quantitect RT mix (Qiagen), 0.5 $\mu$ l TaqMan probe and primers (Life Technologies), and $100 \mathrm{ng}$ total RNA. Thermal cycling conditions comprised an initial step at $95^{\circ} \mathrm{C}$ for $10 \mathrm{~min}$, followed by 45 cycles at $95^{\circ} \mathrm{C}$ for $10 \mathrm{sec}, 60^{\circ} \mathrm{C}$ for $10 \mathrm{sec}$ and $72^{\circ} \mathrm{C}$ for $5 \mathrm{sec}$. The TaqMan ${ }^{\circledR}$ probe and primers for interferon response genes, interferon stimulated gene factor $3 \gamma$ (ISGF-3 $\gamma$ ), 2', 5'-oligoadenylate synthetase 2 (OAS2), interferon-induced myxovirus resistance protein 1 (MX1) and HMBS were purchased from Life Technologies. The 5'-fluorescent reporter dye fluorescence was detected using a LightCycler (Roche Diagnostics).

Statistical analysis. All in vitro experiments were performed in triplicate and repeated 3 times. Student's t-test was used to determine the significance of differences between the groups, with values of $\mathrm{P}<0.05$ considered statistically significant.

\section{Results}

Overexpression of Aktl in human OSCC cells and tissues. Using Human Genome Survey Arrays, we determined the gene expression profiles in 10 primary OSCC tissues, 10 human OSCC cell lines, 3 normal oral mucosal tissues and a human immortalized non-neoplastic keratinocyte cell line. We evaluated gene expression relative to the signal to noise $(\mathrm{S} / \mathrm{N})$ ratio, with an $\mathrm{S} / \mathrm{N}$ ratio $<3$ interpreted as lack of gene expression and an $\mathrm{S} / \mathrm{N}$ ratio $>3$ interpreted as positive gene expression. The expression of 7,417 genes was undetectable in all normal oral mucosal tissues and in a non-neoplastic epithelial cell line. The only gene we identified as expressed in all OSCC tissues and cultured cells was Akt1 (Fig. 1A).

To confirm the microarray data, we examined the expression of Akt1 protein in human OSCC cells and tissues by western blot analysis. All human OSCC cells expressed Akt1 protein at a much higher level than the non-neoplastic epithelial cell line, HaCaT (Fig. 1B). Subsequently, we compared 
A

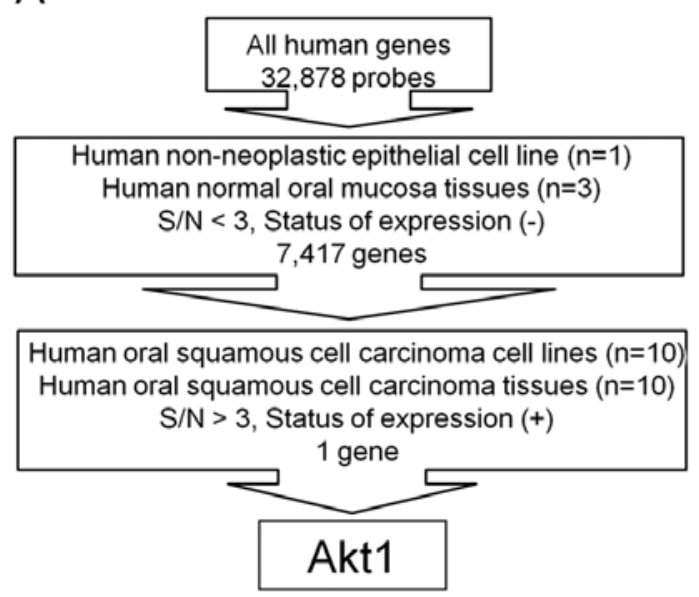

B

Akt1

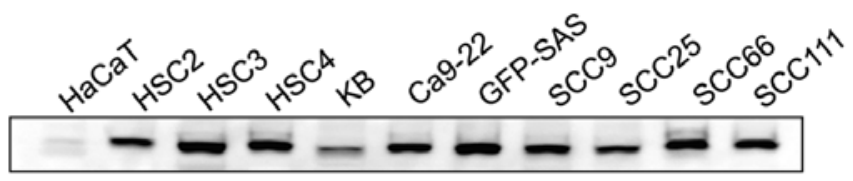

ß-tubulin

C

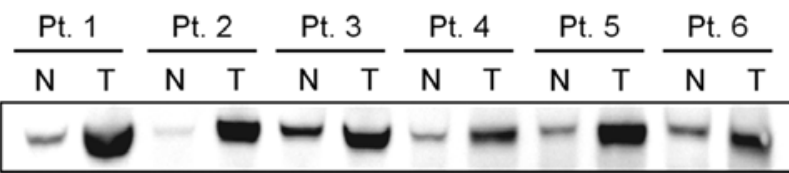

$\beta$-tubulin

Figure 1. Overexpression of Akt1 in oral squamous cell carcinoma (OSCC). (A) Experimental plan for microarray analysis. Using Human Genome Survey Arrays, we determined the gene expression profiles of 10 primary OSCC tissues, 10 human OSCC cell lines, 3 normal oral mucosa tissues, and an immortalized non-neoplastic keratinocyte cell line, HaCaT. The status of gene expression was evaluated relative to the signal to noise (S/N) ratio, with a ratio of 3 and above interpreted as positive expression. In all normal oral mucosal tissues and a non-neoplastic epithelial cell line, 7,417 genes were not expressed. Only Akt1 was expressed in all OSCC tissues and cell lines. (B) Western blots showing the expression of Akt1 protein in all human OSCC cell lines. (C) Western blots showing the overexpression of Akt1 protein in primary tumor tissues $(\mathrm{T})$ compared with adjacent normal tissues $(\mathrm{N})$ from the same $\mathrm{OSCC}$ patient $(\mathrm{Pt})$.

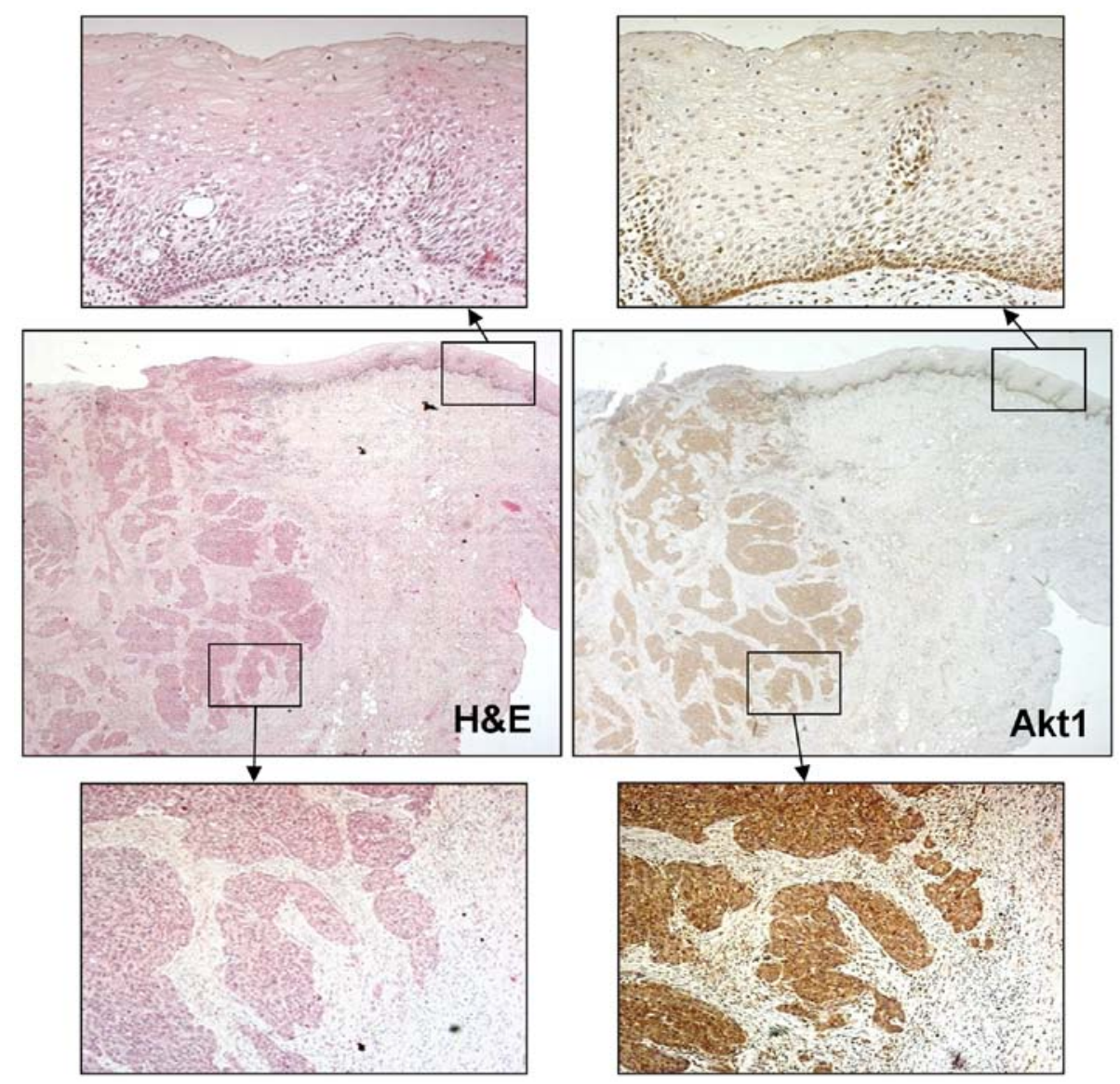

Figure 2. Immunohistochemical staining of Akt1 protein in OSCC tissues. Left hand panels show a HE-stained section and right hand panels show a section stained for Aktl immunohistochemically. Bottom panels show that diffusely positive staining for Akt is focused in cancer cells. Top panels show normal epithelial cells are negative for cytoplasmic Akt1 expression.

the expression levels of Akt1 protein in primary OSCCs with adjacent normal oral mucosal tissues from the same patient and found that Akt1 protein was more highly expressed in the tumor tissues than in the normal tissues (Fig. 1C). Furthermore, immunohistochemical examination showed the expression of Akt1 protein in 59 of 63 (94\%) primary OSCC tissues. Akt1 
A

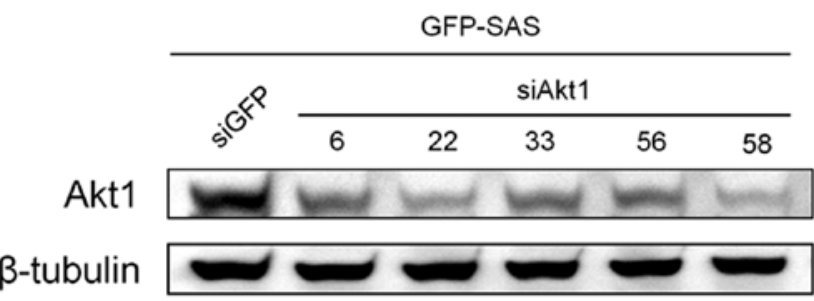

B

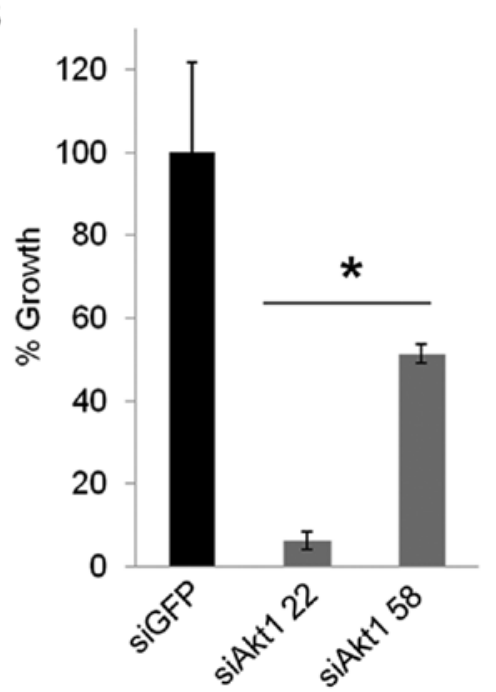

C

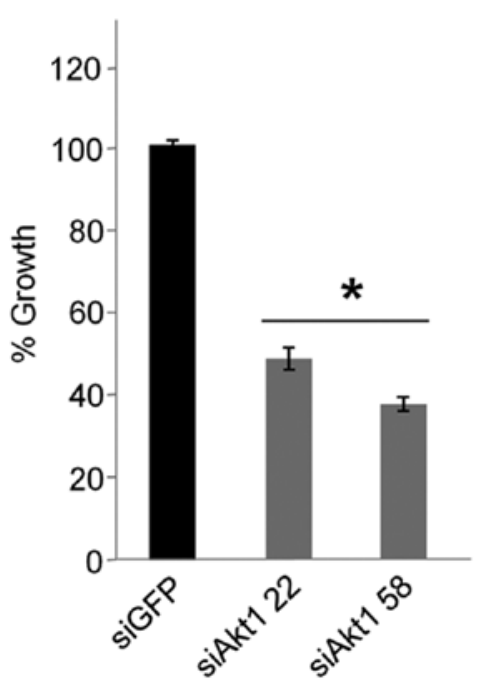

Figure 3. Effects of Akt1 knockdown in the human OSCC cell line, GFP-SAS. (A) Five siAkt1 constructs (siAkt1-6, siAkt1-22, siAkt1-33, siAkt1-56, and siAkt1-58) were transfected into cells at $10 \mathrm{nM}$ with Lipofectamine RNAiMAX. The effects on Akt1 protein expression were evaluated by western blotting and showed that all siAkt1 constructs suppressed expression. (B) The effects of siAkt1-22 and 58 on cell growth were assessed in cells seeded in complete medium and both siRNA constructs significantly inhibited cell growth. (C) The effects of siAkt1-22 and 58 on the invasive growth were examined in the collagen gel culture system and they significantly suppressed the invasive growth. ${ }^{*}<0.01$ compared to control cultures transfected with siGFP.

staining was diffusely positive in OSCCs but not in normal epithelium (Fig. 2). These results suggested that Akt1 was commonly overexpressed in OSCC.

Effect of siAkt1 on the growth of human OSCC cells in vitro. We designed five siRNAs, siAkt1-6, 22, 33, 56 and 58, and transfected them into GFP-SAS cells at a concentration of $10 \mathrm{nM}$, to avoid off-target effects and interferon responses. Synthetic siAkt1-22 and 58 had potent RNA interference (RNAi) effects (Fig. 3A). When the effects of these two siRNAs on cell growth were tested in human OSCC cells, we found that the knockdown of Akt1 expression by siAkt1-22 and 58 significantly inhibited the growth of GFP-SAS cells by between 49 and $94 \%$ compared with the control, siGFP (Fig. 3B). We also examined their effects on the invasive growth of OSCC cells using the collagen gel culture system. Both siAkt1-22 and 58 reduced the invasive growth of GFP-SAS cells by 52 and $63 \%$, respectively (Fig. 3C).

Effect of siAktl on the in vivo growth of human OSCC cells. We assessed the growth inhibitory effect of siAkt1 in vivo using a mouse model. We selected GFP-SAS cells for the in vivo assay because, of the human OSCC cells we used, only these cells showed stable tumorigenicity. We administered siAkt1/atelocollagen complexes into mouse tail veins every 3 days, on 9 occasions. We found that these complexes markedly reduced the size of subcutaneously xenografted GFP-SAS tumors, compared with the siGFP controls (Fig. 4A). During the administration of siAkt1, no reduction in food intake or body weight was observed in the mice.

Only siAkt1-22 can target both human and mouse Akt1. The expression of Akt1 in excised tumor tissues was markedly suppressed by $58 \%$ in the groups injected with siAkt1-22/ atelocollagen complex systemically, but Akt1 expression levels in the lung, liver and kidney did not change (Fig. 4B). We also checked whether the systemic delivery of atelocollagenmediated siRNA induced interferon responses in mice. After injecting the siRNA/atelocollagen i.v., we evaluated mRNA expression of the interferon response genes, ISGF $3 \gamma, \mathrm{OAS} 2$, and MX1, in lung, liver and kidney by qRT-PCR. We compared this to a positive control group injected with poly(I:C), which induced a strong interferon response, but the siRNAs did not (Fig. 5). These results indicated that atelocollagen specifically delivered siRNAs into tumor tissues without inducing an interferon response.

Effect of siAkt1 on the growth of human OSCC primary cultured cells. To confirm the usefulness of targeting Akt1 in OSCC, we used primary cultured cells obtained from resected tumors from four OSCC patients. Two of these primary cultured 
A

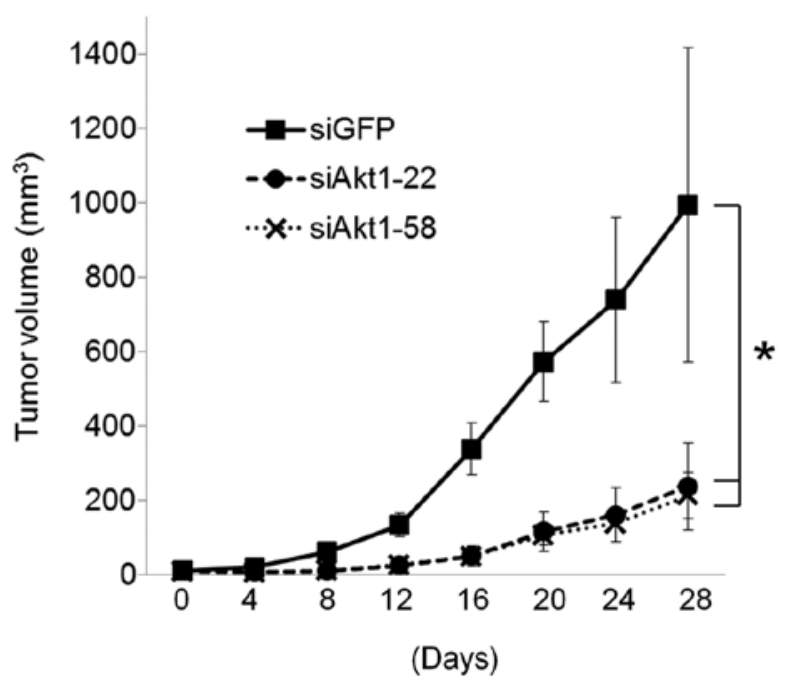

B
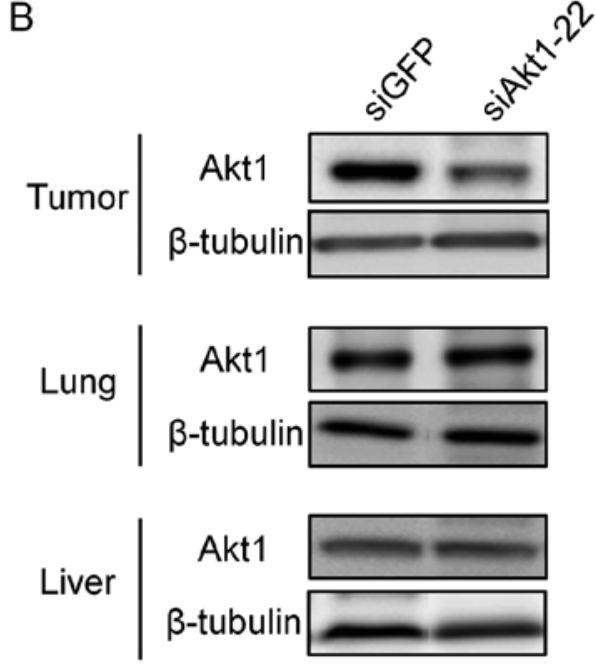

Kidney $\mid \begin{gathered}\text { Akt1 } \\ \beta \text {-tubulin }\end{gathered}$

Figure 4. Effects of Akt1 knockdown in OSCC xenografts. (A) GFP-SAS cells (1x106/50 $\mu 1)$ with an equal volume of Matrigel were injected subcutaneously into the flanks of nude mice. The mice were then injected i.v. with siAkt1/atelocollagen complexes every 3 days. The growth of GFP-SAS tumors in vivo was significantly reduced $(n=6),{ }^{*} \mathrm{p}<0.01$ compared to control group treated with siGFP. (B) Western blots showing the expression levels of Akt1 protein in excised tissues. Treatment with siAktl reduced the expression of Akt1 protein in the tumor tissues but not in lung, liver and kidney.
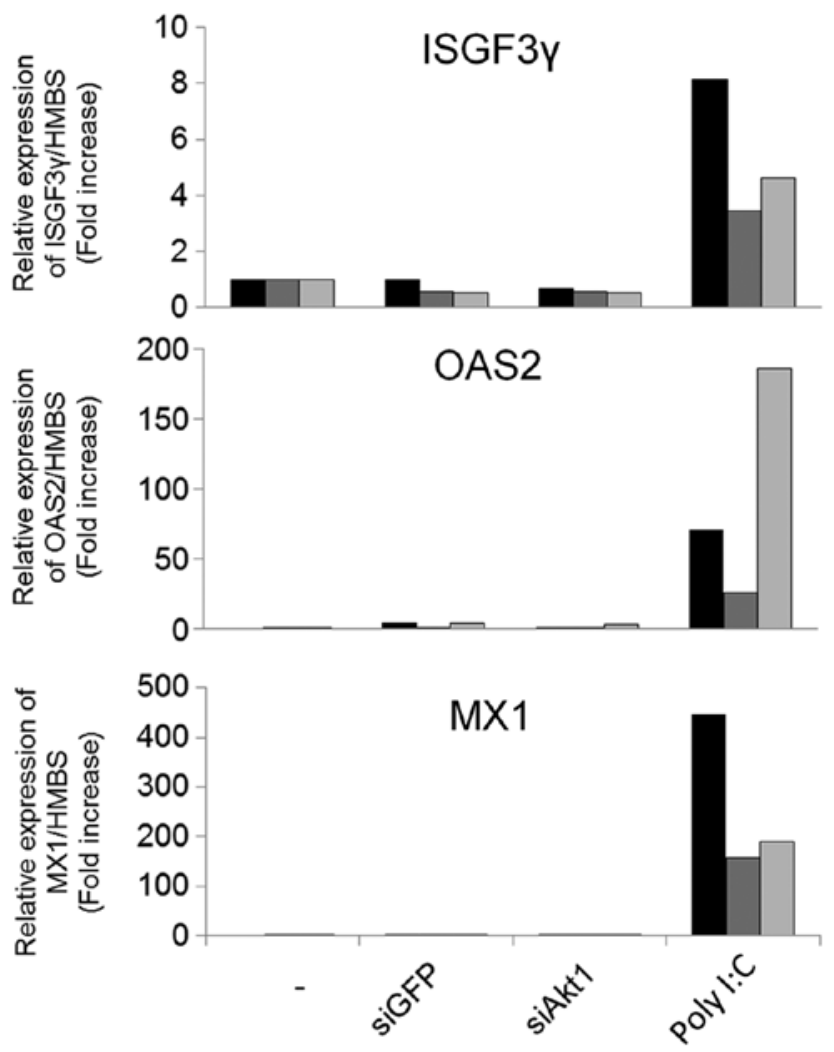

Lung

Liver

Kidney

Figure 5. Interferon responses to atelocollagen-mediated siRNA systemic delivery. After i.v. injection of siRNA/atelocollagen complexes into nude mice, mRNA expression of the interferon response genes ISGF3 $\gamma$, OAS2, and MX1 was evaluated in lung, liver and kidney by qRT-PCR. Poly(I:C) was used as a positive control. Although poly(I:C) induced a strong response, siRNA/atelocollagen did not. cells were derived from tumors of the tongue and two from lower gingival tumors. We then examined the in vitro effects of siAktl on the growth of these primary cultured OSCC cells. As in GFP-SAS cells, transfecting $10 \mathrm{nM}$ siAkt1-22 or 58 into these primary cell cultures reduced Akt1 expression and significantly suppressed cell growth, by between 37 and $76 \%$, compared with siNT (Fig. 6A and B).

Molecular mechanisms of the anti-tumor effects of Akt1 knockdown in human OSCC cells. To understand the molecular mechanisms involved in the growth inhibitory effect of siAkt1, we carried out an oligonucleotide-based microarray analysis after transfecting siAkt1 into GFP-SAS cells. A total of 23 genes were differentially upregulated by $>3$-fold in GFP-SAS cells transfected with either siAkt1-22 or 58, whereas 82 were differentially downregulated by $>3$-fold. Among these genes, cancer-related genes were identified by functional analysis using IPA software (Table I). Akt1 knockdown induced the expression of cyclin-dependent kinase inhibitor 2B (CDKN2B, $\mathrm{p} 15)$, a tumor suppressor gene, and reduced the expression of transforming growth factor, $\beta$ receptor 1 (TGFBR1), which supports malignant phenotypes.

\section{Discussion}

In microarray analysis, we identified Akt1 as the only candidate gene commonly overexpressed in OSCC tissues and cell lines. Overexpression of Akt1 protein in OSCC was also confirmed by western blot analysis and immunohistochemistry. Furthermore, targeting Akt1 by RNAi significantly suppressed the growth of human OSCC cells in vitro and in vivo. These data suggest that Akt1 functions as a critical 


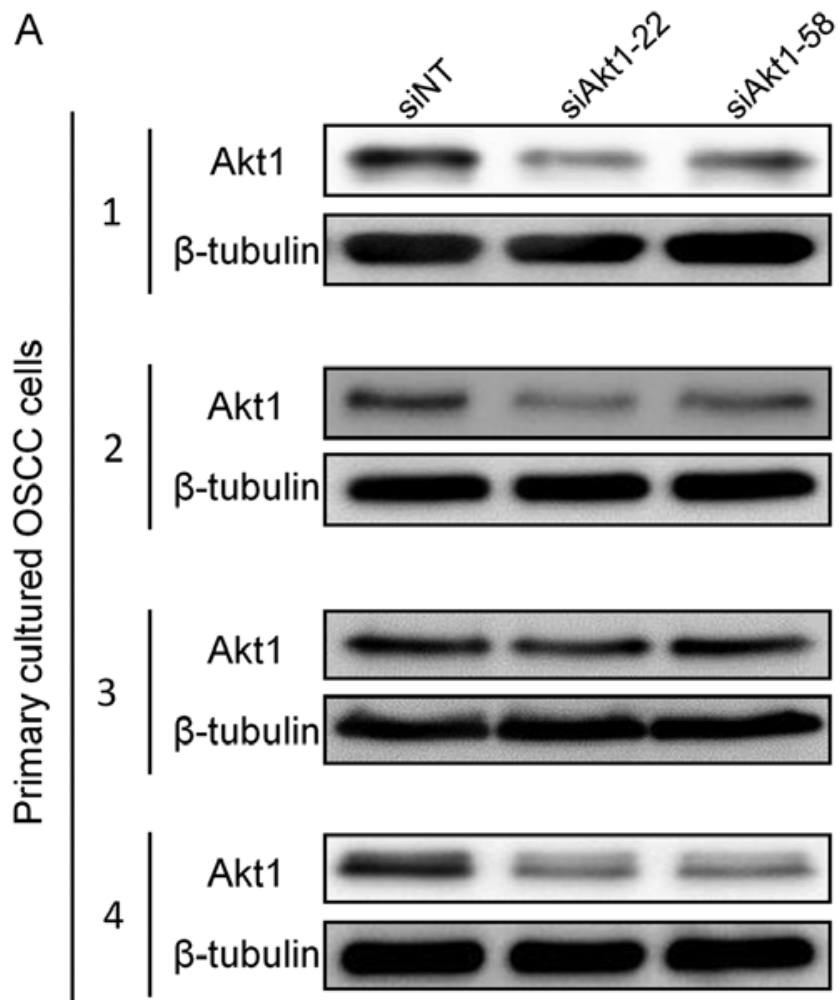

B

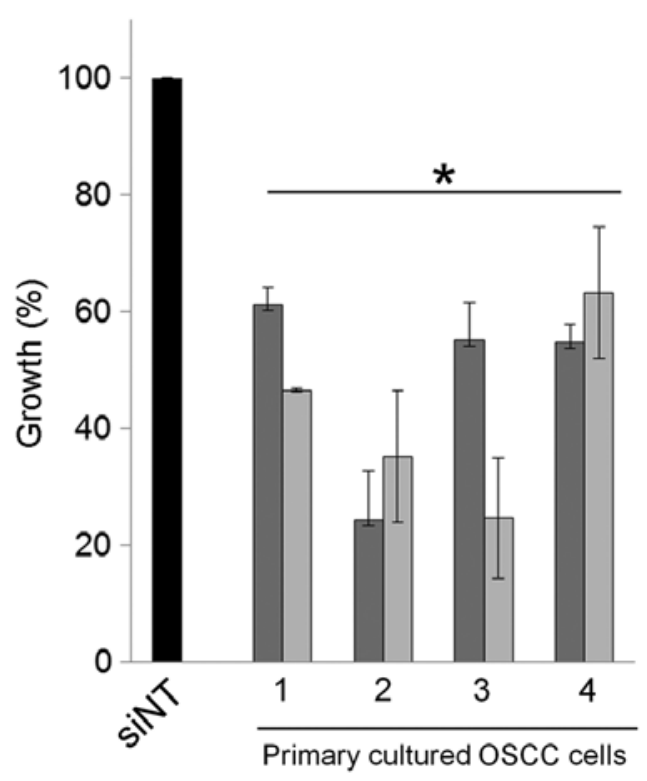

Figure 6. Effect of targeting Akt1 in primary cultures of OSCC cells. (A) Primary cell cultures derived from OSCC tumors from 4 patients were transfected with siAkt1, cultured for $48 \mathrm{~h}$, and lysed. The inhibitory effects of siAkt1-22 and -58 on Akt1 expression were confirmed by western blotting. (B) Primary cell cultures were transfected with $10 \mathrm{nM}$ siAkt1 in Lipofectamine RNAiMAX. Cell growth was evaluated after 3 days using WST-8 assays. Knockdown of Akt1 expression inhibited the growth of OSCC primary cell culture, ${ }^{*} \mathrm{p}<0.01$ compared to control cultures treated with siNT.

Table 1. Cancer-related genes regulated by Akt1 knockdown in GFP-SAS cells.

Gene symbol Gene name Fold change

Up-regulated genes in Akt1 knockdown cells

TXNIP

CDKN2B

PVRL4

VAV3

Down-regulated genes in Akt1 knockdown cells

CDC6
NFIB
IL6R
AHNAK2
FST
BLM
CTGF
MYEOV
PLAGL1
IGFBP3
SOCS2
LIFR
IL7R
AKT1
TGFBR1

Thioredoxin interacting protein

Cyclin-dependent kinase inhibitor 2B

5.475

Poliovirus receptor-related 4

4.389

Vav 3 guanine nucleotide exchange factor

3.019

Cell division cycle 6

Nuclear factor I/B

$-3.263$

Interleukin 6 receptor

$-3.282$

$-3.350$

AHNAK nucleoprotein 2

$-3.401$

Bloom syndrome, RecQ helicase-like

$-3.767$

Connective tissue growth factor

$-4.137$

$-4.422$

$-4.552$

$-4.689$

$-4.942$

$-6.629$

$-7.411$

$-8.461$

$-14.621$ 
oncogene in OSCC and may be an appropriate therapeutic target for patients with OSCC.

The proto-oncogene Akt, also known as protein kinase B, is a well-characterized serine/threonine kinase that lies downstream of phosphatidylinositol 3-kinase (PI3K). In many human malignancies, activated Akt promotes cell proliferation, survival and metastasis of cancer cells by regulating the activity of a number of downstream molecules, such as mammalian target of rapamycin (mTOR), glycogen synthase kinase 3 (GSK3) and Bcl-2-associated death promoter (BAD) $(15,16)$. Akt activation has also been reported to be a significant prognostic indicator in OSCC, with its inhibition being a possible molecular approach to treatment (17). MK-2206 is a highly potent, allosteric pan-Akt inhibitor. In vitro, MK-2206 has shown synergistic inhibitory effects in combination with paclitaxel in HNSCC cell lines. In a phase I clinical trial of MK-2206 in combination with carboplatin/paclitaxel, in patients with advanced solid tumors, there was a partial response in 1 patient with HNSCC and disease was stabilized for $>6$ months in 6 patients (18).

Mammals have three homologous Akt serine/threonine kinases, known as Akt1, Akt2 and Akt3. The phenotypes of knockout mice have suggested a critical role for Akt1 in cell survival, a role for Akt 2 in glucose metabolism and a role for Akt3 in brain development (19). In cancers, overexpression of specific Akt isoforms, with or without gene amplification, has been reported. For example, upregulation has been seen in the expression of Akt1 in gastric and breast cancers, of Akt 2 in ovarian, pancreatic, liver, and colorectal cancers, and of Akt3 in melanoma and breast cancers. Furthermore, a somatic activating mutation in Akt1 has been identified in ovarian, breast, and colorectal cancers. Although the correlation between the development of specific cancers and the hyperactivation of specific Akt isoforms remains unclear, a deficiency of Akt1 has been shown to be sufficient to inhibit endometrial and prostatic neoplasia in phosphatase and tensin homolog (PTEN) mice (19).

Whole-exome sequencing data from HNSCC primary tumors has shown that $30.5 \%$ of mutations were in the PI3K pathway, the most frequently mutated oncogenic pathway. Phosphatidylinositol-4,5-bisphosphate 3-kinase, catalytic subunit $\alpha$ (PIK3CA) was identified as the most commonly mutated gene $(12.6 \%)$ in the PI3K pathway, but mutations in the Akt1 gene were not detected (20). In OSCC, mutational analysis of the PI3K pathway has also shown that missense mutations were only found in the PIK3CA gene $(10.8 \%)$, but not in the Akt1 or PTEN genes (21). Patient-derived tumorgrafts with PI3KCA mutations growing in nonobese diabetic/severe combined immunodeficient $\gamma$ (NOD/SCID $\gamma)$ mice were sensitive to BEZ-235, an mTOR/PI3K inhibitor, whereas PIK3CA-wild-type tumorgrafts were not. Xenografts developed from an HNSCC cell line harboring a PIK3CA mutation were more sensitive to a combination of BEZ-235 plus cetuximab than to cetuximab alone (20). Most recently, Naruse and colleagues reported that phosphorylated mTOR was overexpressed in approximately half of OSCC cases and that its expression level was correlated with the TN classification and survival rate. Furthermore, everolimus, an mTOR inhibitor, suppressed the growth, invasion and migration of human OSCC cells (22).
Our study has also shown that atelocollagen-mediated, systemic administration of siAkt1 suppressed the expression of Akt1 in tumor tissues but not in normal tissues and the growth of human OSCC cells in nude mice, without severe side effects such as lung, liver, or renal damage. This selective targeting may be due to the vascular abnormalities of tumor tissues, called the enhanced permeability and retention (EPR) effect (23). Furthermore, atelocollagen is a highly biocompatible biomaterial having been used in the field of healthcare as a component of local hemostatic agents and for tissue regeneration and is unlikely to induce immune reactions in vivo. These results suggest that nucleic acid-based drugs, such as atelocollagen-complexed siRNA, may provide novel therapeutic opportunities for human malignancies, with minimal risks of adverse events.

In conclusion, overexpression of Akt1 plays a critical role in OSCC progression and targeting the PI3K/Akt/mTOR pathway appears an appropriate approach for novel therapies for HNSCC, including OSCC.

\section{Acknowledgements}

This study was supported by Grant-in-Aid for Young Scientists (A) 17689057 and Scientific Research (C) 21592557 in Japan Society for the Promotion of Science.

\section{References}

1. Ferlay J, Shin HR, Bray F, Forman D, Mathers C and Parkin DM: Estimates of worldwide burden of cancer in 2008: GLOBOCAN 2008. Int J Cancer 127: 2893-2917, 2010.

2. Gupta S, Kong W, Peng Y, Miao Q and Mackillop WJ: Temporal trends in the incidence and survival of cancers of the upper aerodigestive tract in Ontario and the United States. Int J Cancer 125: 2159-2165, 2009.

3. Weinstein IB, Joe A and Felsher D: Oncogene addiction. Cancer Res 68: 3077-3080, 2008

4. Huang SF, Cheng SD, Chien HT, Liao CT, Chen IH, Wang HM, Chuang WY, Wang CY and Hsieh LL: Relationship between epidermal growth factor receptor gene copy number and protein expression in oral cavity squamous cell carcinoma. Oral Oncol 48: 67-72, 2012.

5. Bonner JA, Harari PM, Giralt J, Azarnia N, Shin DM, Cohen RB, Jones CU, Sur R, Raben D, Jassem J, et al: Radiotherapy plus cetuximab for squamous-cell carcinoma of the head and neck. N Engl J Med 354: 567-578, 2006.

6. Vermorken JB, Mesia R, Rivera F, Remenar E, Kawecki A, Rottey S, Erfan J, Zabolotnyy D, Kienzer HR, Cupissol D, et al: Platinum-based chemotherapy plus cetuximab in head and neck cancer. N Engl J Med 359: 1116-1127, 2008.

7. Shintani S, Mihara M, Nakahara Y, Aida T, Tachikawa $T$ and Hamakawa H: Lymph node metastasis of oral cancer visualized in live tissue by green fluorescent protein expression. Oral Oncol 38: 664-669, 2002.

8. Klosek SK, Nakashiro K, Hara S, Goda H and Hamakawa H: Stat 3 as a molecular target in RNA interference-based treatment of oral squamous cell carcinoma. Oncol Rep 20: 873-878, 2008.

9. Shintani S, Hamakawa H, Nakashiro K, Shirota T, Hatori M, Tanaka M, Kuroshita Y and Kurokawa Y: Friend leukaemia insertion (Fli)-1 is a prediction marker candidate for radiotherapy resistant oral squamous cell carcinoma. Int J Oral Maxillofac Surg 39: 1115-1119, 2010.

10. Mu P, Nagahara S, Makita N, Tarumi Y, Kadomatsu K and Takei Y: Systemic delivery of siRNA specific to tumor mediated by atelocollagen: Combined therapy using siRNA targeting Bcl-xL and cisplatin against prostate cancer. Int J Cancer 125: 2978-2990, 2009.

11. Ochiya T, Takahama Y, Nagahara S, Sumita Y, Hisada A, Itoh H, Nagai Y and Terada M: New delivery system for plasmid DNA in vivo using atelocollagen as a carrier material: The Minipellet. Nat Med 5: 707-710, 1999. 
12. Minakuchi Y, Takeshita F, Kosaka N, Sasaki H, Yamamoto Y, Kouno M, Honma K, Nagahara S, Hanai K, Sano A, et al: Atelocollagen-mediated synthetic small interfering RNA delivery for effective gene silencing in vitro and in vivo. Nucleic Acids Res 32: e109, 2004.

13. Azuma K, Nakashiro K, Sasaki T, Goda H, Onodera J, Tanji N, Yokoyama M and Hamakawa H: Anti-tumor effect of small interfering RNA targeting the androgen receptor in human androgen-independent prostate cancer cells. Biochem Biophys Res Commun 391: 1075-1079, 2010.

14. Sasaki T, Nakashiro K, Tanaka H, Azuma K, Goda H, Hara S, Onodera J, Fujimoto I, Tanji N, Yokoyama M, et al: Knockdown of Akt isoforms by RNA silencing suppresses the growth of human prostate cancer cells in vitro and in vivo. Biochem Biophys Res Commun 399: 79-83, 2010.

15. Luo J, Manning BD and Cantley LC: Targeting the PI3K-Akt pathway in human cancer: Rationale and promise. Cancer Cell 4 257-262, 2003.

16. Vivanco I and Sawyers CL: The phosphatidylinositol 3-kinase AKT pathway in human cancer. Nat Rev Cancer 2: 489-501, 2002.

17. Lim J, Kim JH, Paeng JY, Kim MJ, Hong SD, Lee JI and Hong SP: Prognostic value of activated Akt expression in oral squamous cell carcinoma. J Clin Pathol 58: 1199-1205, 2005.
18. Beck JT, Ismail A and Tolomeo C: Targeting the phosphatidylinositol 3-kinase (PI3K)/AKT/mammalian target of rapamycin (mTOR) pathway: An emerging treatment strategy for squamous cell lung carcinoma. Cancer Treat Rev 40: 980-989, 2014.

19. Gonzalez E and McGraw TE: The Akt kinases: Isoform specificity in metabolism and cancer. Cell Cycle 8: 2502-2508, 2009.

20. Lui VWY, Hedberg ML, Li H, Vangara BS, Pendleton K, Zeng Y, Lu Y, Zhang Q, Du Y, Gilbert BR, et al: Frequent mutation of the PI3K pathway in head and neck cancer defines predictive biomarkers. Cancer Discov 3: 761-769, 2013.

21. Cohen Y, Goldenberg-Cohen N, Shalmon B, Shani T, Oren S, Amariglio N, Dratviman-Storobinsky O, ShnaidermanShapiro A, Yahalom R, Kaplan I, et al: Mutational analysis of PTEN/PIK3CA/AKT pathway in oral squamous cell carcinoma. Oral Oncol 47: 946-950, 2011.

22. Naruse T, Yanamoto S, Yamada S, Rokutanda S, Kawakita A, Kawasaki G and Umeda M: Anti-tumor effect of the mammalian target of rapamycin inhibitor everolimus in oral squamous cell carcinoma. Pathol Oncol Res 21: 765-773, 2015.

23. Takeshita F, Minakuchi Y, Nagahara S, Honma K, Sasaki H, Hirai K, Teratani T, Namatame N, Yamamoto Y, Hanai K, et al: Efficient delivery of small interfering RNA to bone-metastatic tumors by using atelocollagen in vivo. Proc Natl Acad Sci USA 102: 12177-12182, 2005. 